\title{
Spatial Patterns of Prisoner's Dilemma Game in Metapopulations
}

\author{
C. Huia ${ }^{a, *}$ M. A. McGeoch ${ }^{\mathrm{b}}$ \\ ${ }^{a}$ Spatial, Physiological and Conservation Ecology Group, Department of Entomology, \\ University of Stellenbosch, Private Bag X1, Matieland 7602, South Africa \\ ${ }^{b}$ Center of Invasion Biology, Department of Entomology, University of Stellenbosch, \\ Private Bag X1, Matieland 7602, South Africa
}

Received: 19 July 2005 / Accepted: 30 May 2006 / Published online: 29 July 2006 (c) Society for Mathematical Biology 2006

\begin{abstract}
Because to defect is the evolutionary stable strategy in the prisoner's dilemma game (PDG), understanding the mechanism generating and maintaining cooperation in PDG, i.e. the paradox of cooperation, has intrinsic significance for understanding social altruism behaviors. Spatial structure serves as the key to this dilemma. Here, we build the model of spatial PDG under a metapopulation framework: the sub-populations of cooperators and defectors obey the rules in spatial PDG as well as the colonization-extinction process of metapopulations. Using the mean-field approximation and the pair approximation, we obtain the differential equations for the dynamics of occupancy and spatial correlation. Cellular automaton is also built to simulate the spatiotemporal dynamics of the spatial PDG in metapopulations. Join-count statistics are used to measure the spatial correlation as well as the spatial association of the metapopulation. Simulation results show that the distribution is self-organized and that it converges to a static boundary due to the boycotting of cooperators to defectors. Metapopulations can survive even when the colonization rate is lower than the extinction rate due to the compensation of cooperation rewards for extinction debt. With a change of parameters in the model, a metapopulation can consist of pure cooperators, pure defectors, or cooperator-defector coexistence. The necessary condition of cooperation evolution is the local colonization of a metapopulation. The spatial correlation between the cooperators tends to be weaker with the increase in the temptation to defect and the habitat connectivity; yet the spatial correlation between defectors becomes stronger. The relationship between spatial structure and the colonization rate is complicated, especially for cooperators. The metapopulation may undergo a
\end{abstract}

\footnotetext{
*Corresponding address.

E-mail address: chui@sun.ac.za (C. Hui).
} 
temporary period of prosperity just before the extinction, even while the colonization rate is declining.

Keywords Cooperation · Game theory · Join-count statistics · Pair approximation - Spatial correlation and association

\section{Introduction}

The evolution of altruistic behavior is a fundamental issue in evolutionary biology. Darwin's natural selection is based on individual selection; however, altruistic behavior obviously means cost to its actor. In searching for the approach through which altruistic behavior emerges and persists, scientists have generally presented three theories: group selection (Williams, 1966; Wilson and Sober, 1994), kin selection (Hamilton, 1964), and reciprocal altruism (Trivers, 1971; Axelrod and Hamilton, 1981). The group selection indicates that natural selection acts on organism groups, rather than on individuals. Kin selection suggests that most of the benefit of altruism goes to relatives and therefore benefits the gene of altruism (Hamilton, 1964). The theory of reciprocal altruism based on prisoner's dilemma games (PDG) is adopted by most evolutionary biologists to explain the evolution of altruistic behavior (Harms, 1999; Hoffmann, 2000; Nowak and Sigmund, 2004).

In the PDG, each of two players has to decide simultaneously and independently to cooperate, $C$, or defect, $D$ (Nowak and May, 1992). The payoff to a player depends on its own decision-making and also the choice of the opponent. If both players choose $C$, both get a payoff of magnitude $R$ (the reward for mutual cooperation); if one defects while the other cooperates, $D$ gets the game's biggest payoff, $T$ (the temptation to defect), while $C$ gets $S$ (the payoff of sucker); if both defect, both get $U$ (the punishment for mutual defect) (Dieckmann et al., 2000). The payoff is also typically expressed in a matrix form,

$$
\begin{gathered}
C \\
C \\
D
\end{gathered}\left[\begin{array}{ll}
R & S \\
T & U
\end{array}\right] .
$$

The prisoner's dilemma is defined by the inequalities $T>R>U>S>$ and $2 R>$ $T+S$. Since defect behavior is an evolutionary stable strategy (ESS) and Nash equilibrium in the PDG (Axelrod and Hamilton, 1981), the successful invasion of the cooperators into defectors, is almost impossible; even the resistance of cooperators to defector's invasion becomes difficult. Organisms look forward to the reward from the recipient even though the altruistic behavior incurs cost to the organism itself, such as the reciprocal feeding in vampire bats (Wilkinson, 1984). This incurs the paradox of cooperation (how the altruistic behavior is maintained in the natural world), especially for social species. The efforts to break this deadlock lead to the construction of complex strategies based on the iterated prisoner's dilemma games, such as the famous behavior strategy Tit-for-Tat (Nowak and Sigmund, 1993). 
Although the importance of spatial interactions has been recognized for many years, it is only recently (Levin et al., 1997), with the utilization of new techniques and the availability of more powerful computers, that spatially explicit dynamics have begun to be considered by ecologists. The progress in spatial ecology (Tilman and Kareiva, 1997), especially of the knowledge in metapopulation processes, i.e. a colonization-extinction dynamics (Hanski, 1998), enabled scientists to answer many open problems in ecology. For example, the resolving of Hutchinson's (1961) paradox (species coexistence in homogeneous habitat vs. competition exclusive principle) and Fisher's (1930) paradox (polymorphism vs. genetic load) heavily relies on the spatial and metapopulation structure of species (Hanski and Zhang, 1993; Tilman, 1994; Hui and Yue, 2005). The metapopulation framework has become an essential instrument for modeling the ecological and evolutionary dynamics of a spatially structured habitat (e.g. Hanski, 1998; Hui and Li, 2003; Hanski and Gaggiotti, 2004).

Spatial models have also been introduced into the PDG to resolve the paradox of cooperation. The paradigm of spatial PDG is as follows (Nowak and May, 1992): the game occurs between local neighbors, where a high fitness individual excels. When mortality occurs, a neighbor will be randomly chosen to replace the empty patch immediately. It focuses on the dynamics of the relative frequency of each behavioral strategy within a constant population (Nowak et al., 1994; Nowak and Sigmund, 2004). Yet, two issues can not be addressed: (1) the effect of PDG on the total population dynamics as there are no empty patches in the traditional spatial PDG; (2) the effect of colonization-extinction dynamics as well as PDG on the spatial structure of the total species for the same reason. Note, here we only discuss the intraspecific altruism, which means that the cooperator and the defector are only the individual/sub-population of one species. In this work, we will try to build up the spatial PDG in a metapopulation framework. Although some works have already studied the PDG in a metapopulation framework (Harms, 1999), the focus, simulation scale, as well as model type of this work are quite different. Using mathematical analysis and computer simulation, we will present (1) the primary factors contributing to the evolution of cooperation, (2) the spatiotemporal dynamics of the metapopulation consisting of defector and cooperator sub-populations using the mean-field approximation as well as the pair approximation (Matsuda et al., 1992; Harada and Iwasa, 1994; Hui and Li, 2004), (3) the spatial structure of the defector, the cooperator and the metapopulation using join-count statistics (JCS) (Upton and Fingleton, 1985; Fortin et al., 2002; Hui et al., 2006).

\section{Model}

Metapopulation describes a 'population' consisting of many local populations (Levins, 1969). All the local populations have a substantial probability of extinction, and hence the long-term persistence of the species can only occur at the regional or metapopulation level (Hanski, 1998). The classical metapopulation framework is based on Levins' (1969) patch occupancy model,

$$
\frac{\mathrm{d} P}{\mathrm{~d} t}=c P(1-P)-e P,
$$


where $P$ is the fraction of patches occupied by the species (i.e. the occupancy), and $c$ and $e$ are the colonization and extinction rate, respectively. The non-trivial equilibrium $\hat{P}=1-e / c$ is globally stable as long as $e<c$ (Tilman and Kareiva, 1997; Hanski, 1999). The Levins model assumes infinite habitat patches but also that colonization is not affected by distance (Hanski, 1999). This assumption means that all patches are equally connected to other patches, which is called the mean-field assumption (Hanski, 1999; Hui and Li, 2003, 2004) and is often a good approximation for metapopulation at a stochastic steady-state even if migration is distance-dependent (Nisbet and Gurney, 1982; Durrett and Levin, 1994).

\subsection{Mean-field approximation}

Now, we introduce PDG into the metapopulation framework. Without losing generality, let $T=b, R=1, U=-1$ and $S=-b(b>1)$, which will not affect the essential characteristics of the PDG (Epstein and Axtell, 1996). Let $P_{C}$ and $P_{D}\left(P_{C}+P_{D}=P\right)$ be the occupancy fractions of the cooperators and the defectors. The mean payoffs of $C$ and $D$ are $w_{C}=P_{C}-b P_{D}$ and $w_{D}=b P_{C}-P_{D}$, respectively. Note that $-b \leq w_{C} \leq 1$ and $-1 \leq w_{D} \leq b$. These payoffs will greatly influence the colonization and local extinction of the metapopulation. As with other classic models of PDG (e.g. Nowak and May, 1992; Dieckmann et al., 2000; Zhang et al., 2005), we assume that the colonization rate of a sub-population is positively correlated with its payoff, while the extinction rate is negatively correlated. Here, we choose the simplest linear relationships as follows:

$$
c_{\Omega}=\alpha \frac{b+w_{\Omega}}{2 b} \text { and } e_{\Omega}=\beta \frac{b-w_{\Omega}}{2 b},
$$

where $\Omega$ is behavior $C$ or $D ; \alpha$ and $\beta$ are two coefficients of colonization and extinction, which are positive-linearly correlated with the colonization and extinction rates. According to (1) and (2), we have the dynamics of cooperator and defector in metapopulation structure,

$$
\frac{\mathrm{d} P_{\Omega}}{\mathrm{d} t}=c_{\Omega} P_{\Omega}(1-P)-e_{\Omega} P_{\Omega}
$$

As we only address a one-round PDG here, the individuals or the sub-populations of the metapopulation will not change their behavior strategy during their lifetime (e.g. Harms, 1999). Although it is also called a spatial colonization model or a spatially explicit population model, we will use the loose definition of metapopulation and still call this spatial population a metapopulation (Chapter 1 in Tilman and Kareiva, 1997). After calculation, the equilibrium of this model is

$$
\hat{P}_{C}=0 ; \hat{P}_{D}=\frac{1}{2}\left(\frac{\beta}{\alpha}+b+1-\sqrt{\left(\frac{\beta}{\alpha}+b+1\right)^{2}+4 b\left(\frac{\beta}{\alpha}-1\right)}\right) .
$$

It means that cooperators cannot stably coexist with defectors in a well-mixed population (Nowak and May, 1992; Lindgren and Nordahl, 1994) and also implies that 
the Levins model with the mean-field assumption is not appropriate for describing the spatial patterns and the PDG. There is no chance for the evolution of cooperation in metapopulations under the mean-field assumption (i.e. well-mixed individuals).

\subsection{Pair approximation}

The most powerful approach to modeling spatially structured population dynamics and local processes in ecology, is the lattice model with cellular automaton (e.g. Hui, 2004), which has been widely adopted in the research of metapopulation dynamics and more general questions of spatial ecology (Tilman and Kareiva, 1997; Hanski and Gaggiotti, 2004), as well as of the spatial PDG (e.g. Nowak and May, 1992; Harms, 1999). A potential problem with these spatially explicit models is that analysis is often restricted to direct computer simulation. In fact, these spatially explicit simulation models can be analyzed by a useful approach, called pair approximation, which was introduced to ecological research by Matsuda et al. (1992). This approximation, first used in statistical physics (Katori and Konno, 1991; Tainaka, 1993), has been carried out for many models of population dynamics of plants and metapopulations (Iwasa et al., 1991; Harada and Iwasa, 1994; Harada et al., 1994; Hui and Li, 2004). Pair approximation, as one of the primary approaches of the moment approximations, is conceptually and mathematically similar to the JCS (Hui et al., 2006).

Here, we will introduce the pair approximation into the study of spatial games. According to the processes of PDG in metapopulations, the dynamics of defectors and cooperators can be described by the pair approximation as follows:

$$
\begin{aligned}
\frac{\mathrm{d} P_{C}}{\mathrm{~d} t}=\alpha & \frac{b+Q_{C / E}-b Q_{D / E}}{2 b} P_{C E}-\beta \frac{b P_{C}-P_{C C}+b P_{D C}}{2 b} \\
\frac{\mathrm{d} P_{D}}{\mathrm{~d} t}=\alpha & \frac{b+b Q_{C / E}-Q_{D / E}}{2 b} P_{D E}-\beta \frac{b P_{D}-b P_{C D}+P_{D D}}{2 b} \\
\frac{\mathrm{d} P_{C C}}{\mathrm{~d} t}= & \frac{\alpha}{b} P_{C E} \nabla_{E C}^{C}\left((1+1 / z) b+\nabla_{E C}^{C}-b \nabla_{E C}^{D}\right) \\
& -\frac{\beta}{b} P_{C C}\left((1-1 / z) b-\nabla_{C C}^{C}+b \nabla_{C C}^{D}\right) \\
\frac{\mathrm{d} P_{D D}}{\mathrm{~d} t}= & \frac{\alpha}{b} P_{D E} \nabla_{E D}^{D}\left((1-1 / z) b+b \nabla_{E D}^{C}-\nabla_{E D}^{D}\right) \\
& -\frac{\beta}{b} P_{D D}\left((1+1 / z) b-b \nabla_{D D}^{C}+\nabla_{D D}^{D}\right) \\
\frac{\mathrm{d} P_{C D}=}{\mathrm{d} t}= & \frac{\alpha}{2 b} \frac{z-1}{z}\left(P_{C E}\left(b+b \nabla_{E C}^{C}-\frac{z-1}{z} Q_{D / E C}\right) Q_{D / E C}\right. \\
& \left.+P_{D E}\left(b+\frac{z-1}{z} Q_{C / E D}-b \nabla_{E D}^{D}\right) Q_{C / E D}\right)
\end{aligned}
$$




$$
\begin{aligned}
& -\frac{\beta}{2 b}\left(P_{C D}\left(b-\frac{z-1}{z} Q_{C / C D}+b \nabla_{C D}^{D}\right)\right. \\
& \left.+P_{C D}\left(b-b \nabla_{D C}^{C}+\frac{z-1}{z} Q_{D / D C}\right)\right)
\end{aligned}
$$

where $P_{\Omega}$ is the probability that a randomly chosen patch is occupied by a $\Omega$ strategy local population (note that $E$ indicates the state of empty patches), $P_{\Omega \Omega}$ is the probability that a randomly chosen pair of nearest-neighbor patches is in state $\Omega \Omega^{\prime}, Q_{\Omega / \Omega^{\prime}}$ is the conditional probability that a randomly chosen neighbor of a patch in state $\Omega^{\prime}$ is in state $\Omega, Q_{\Omega / \Omega^{\prime} \Omega^{\prime \prime}}$ is the conditional probability that a randomly chosen neighbor of the $\Omega^{\prime}$ patch in a $\Omega^{\prime} \Omega^{\prime \prime}$ pair patches is in state $\Omega$, symbol $\nabla_{\Omega^{\prime} \Omega^{\prime \prime}}^{\Omega}$ represents $\left(1+(z-1) Q_{\Omega / \Omega^{\prime} \Omega^{\prime \prime}}\right) / z$, and $z$ is the number of neighbor patches and means the dispersal region of migrants. Equation (5) is analogues to Eq. (3). For a given empty patch, every local population in the habitat can contribute to the colonization of this patch by the mean-field approximation (the term $C_{\Omega} \Omega$ in Eq. (3)), while only local populations in the neighboring patches can colonize the patch by pair approximation (the first right-term in Eq. (5a-d) and the first two right-terms in Eq. (5e)). Noted that $Q_{\Omega /{ }^{\prime} \Omega \Omega^{\prime \prime}}=Q_{\Omega / \Omega^{\prime}}$ in pair approximation and all the probabilities can be calculated by the five variables $\left(P_{C}\right.$, $P_{D}, P_{C C}, P_{D D}$ and $\left.P_{C D}\right)$ in Eq. (5) according to the probability theory $\left(Q_{\Omega / \Omega^{\prime}}=\right.$ $\left.P_{\Omega \Omega^{\prime}} / P_{\Omega^{\prime}}\right)$

\subsection{Spatial analysis}

Two approaches will be adopted for discussing the dynamics and spatial patterns of spatial games in metapopulations. First, we analyze the dynamics and equilibriums of Eq. (5) through the numerical solution (Hilborn, 2000; Hui and Li, 2004) using NDSlove function in Mathemtica (version 5.0.1, Wolfram Inc.). The value of $P_{\Omega}$ and $Q_{\Omega / \Omega^{\prime}}$ at $T=1000$ were assumed to be the equilibriums of the Eq. (5), from which we can obtain all the variables (see below). The second approach is a simulation, with which we can obtain the spatial patterns of PDG (as in Hui and $\mathrm{Li}$, 2003; Hui et al., 2004). We construct a lattice model for a finite number of $n \times n$ patches on a two-dimensional homogeneous patchy habitat with synchronous updating and a von Neumann (four nearest patches) or Moore (eight nearest patches) neighborhood. We used periodic boundaries, which are often used in theoretical literature (Schonfisch, 1997; Kermer et al., 1998; Hui et al., 2004; Sayama, 2004), instead of absorbing boundaries (Huxel and Hastings, 1999). We ignore the time required for movement, which is also the classical assumption in many spatially implicit and explicit models (McGlade, 1999; Dieckmann et al., 2000; Hui and Yue, 2005).

To describe the spatial structure of the distribution pattern, we adopt a spatiostat index, the JCS (Fortin et al., 2002). The JCS is conceptually and mathematically similar to the pair-approximation (or moment approximation) approach used to describe spatial distributions in metapopulation ecology (see Dieckmann et al., 2000 , for outline of the latter). JCS can be used to classify distributions as spatially aggregated, segregated or random in terms of the global and local densities 
used in pair approximation (Sato and Iwasa, 2000; Hui and Li, 2004). Here, we use $I_{\Omega}=Q_{\Omega / \Omega}-P_{\Omega}$ as the specific aggregation index in the model analysis. Spatially explicit aggregation can be described by $I_{\Omega}>0$, indicating the positive first-order spatial correlation between two adjacent, occupied grids. The spatially random distribution has $I_{\Omega}=0$ and implies the independence of two adjacent, occupied grids. Lastly, therefore, the spatially segregated distribution can be depicted by $I_{\Omega}<0$, i.e. a negative spatial correlation between two adjacent samples (Hui and Li, 2004; Hui et al., 2006). The JCS can also be adopted for determining whether and to what extent different species are associated (Cole, 1957; Greig-Smith, 1964; Pielou, 1972; Perry et al., 2002), and therefore for analyzing the association between different behavior strategies. We use $A_{\Omega / \Omega^{\prime}}=Q_{\Omega / \Omega^{\prime}}-Q_{\Omega^{\prime} / \Omega^{\prime}}$ as the index of spatial association. If $A_{\Omega / \Omega^{\prime}}>0$, it means that the behavior $\Omega^{\prime}$ is positively associated with the behavior $\Omega$, i.e. there are more $\Omega$ populations neighboring to $\Omega^{\prime}$ population than $\Omega^{\prime}$ itself. If $A_{\Omega / \Omega^{\prime}}<0$, there is a negative association; if $A_{\Omega / \Omega^{\prime}}=0$, no association is found. Indices $I_{\Omega}$ and $A_{\Omega / \Omega^{\prime}}$ will be adopted to describe the spatial structure of cooperator and defector, as well as the metapopulation, in the spatial PDG.

\section{Results}

From this simulation, we obtain the following results. (1) The cooperators assemble together and are encircled by the defectors (Fig. 1a, von Neumann neighborhood; Fig. 1b, Moore neighborhood). (2) The metapopulation density is obviously higher in the cooperative region than in the defective region (Fig. 1, $t$ test $Q_{C / C}>Q_{D / D} ; t$-value $=100.15, d f=998, p<0.001, z=4 ; t$-value $=9.98$, $d f=879, p<0.001, z=8$ ). (3) The distribution is self-organized since a static border between cooperators and defectors is formed after 75 time steps of the coalescing phase. There are almost no empty patches along the borders in the cooperation side and hence there is no space to let the defectors invade. (4) The effect of the neighboring structure is strong. As the patch connectivity increases (from von Neumann to Moore neighborhood), the clumpy structure breaks down rapidly (Fig. 1). The global density of cooperators with the Moore neighborhood is significantly lower than that with the von Neumann neighborhood ( $t$-value $=66.3$, $d f=998, p<0.001)$. The distribution of cooperators becomes more fragmented in highly connected patchy networks. In addition, in the simulation, the boundary condition (periodic, absorbing, or reflection) does not affect the simulation dynamics, especially when the grid number is high (e.g. no significant difference is found when the grids are more than $50 \times 50$ ).

The simulation also shows the dynamics of the fraction of patches occupied by the cooperators, defectors, as well as the metapopulation (i.e. the cooperators and defectors in total) (Fig. 2a). For the same parameters, the fraction of occupied patches by the metapopulation with a von Neumann neighborhood has higher occupied patches than the one with a Moore neighborhood. The cooperators become dominant in the von Neumann patch network but they become extinct in the Moore patch network (Fig. 2a). The spatial correlation shows a more complicated pattern (Fig. 2b). There is a transitive phase for the aggregation intensities of the 


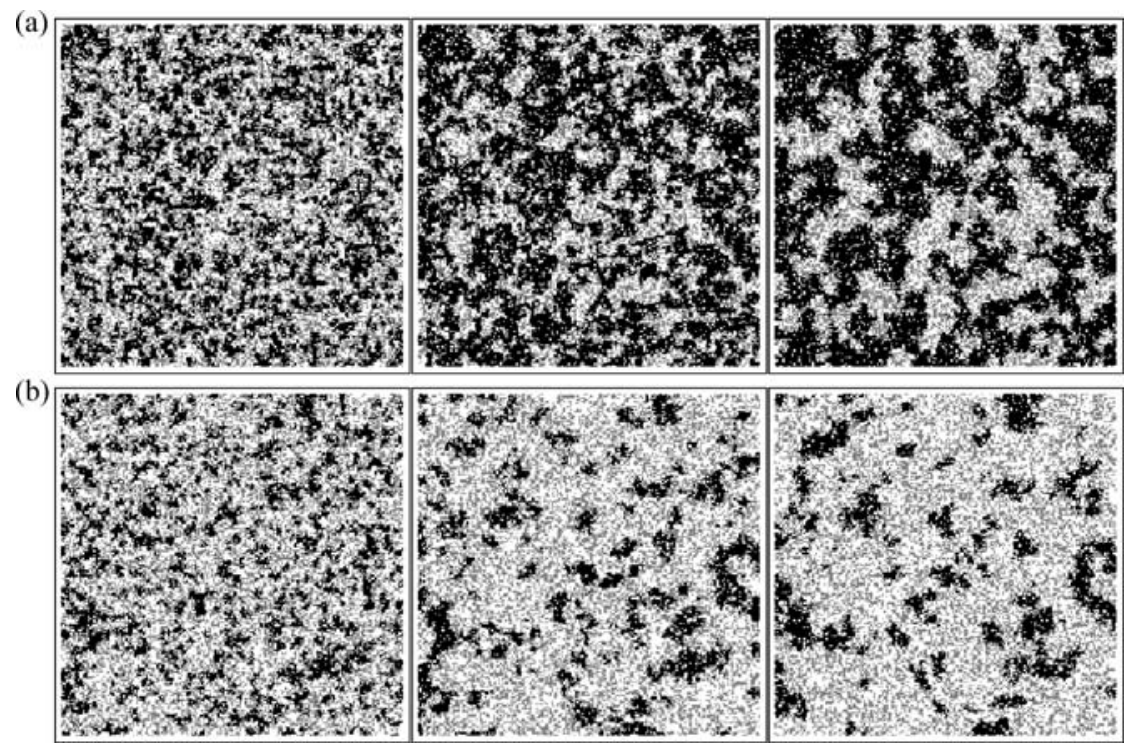

Fig. 1 Spatial dynamics of spatial PDG (a) with von Neumann neighborhood and (b) with Moore neighborhood. Black patches are cooperators, gray patches are defectors, and white patches are empty (in a $200 \times 200$ patches networks at time $t=25,75$ and 125). Parameter values: (a) $\alpha=0.7$, $\beta=0.2, b=1.6, z=4$; (b) $\alpha=0.6, \beta=0.2, b=1.6, z=8$.

cooperator $I_{C}$, the defector $I_{D}$, as well as of the metapopulation $I_{+}\left(I_{+}=Q_{C / D}+\right.$ $Q_{D / C}+I_{C}+I_{D} ; \pm$ indicates the occupied state). The variance of the dynamics of the spatial correlation with the von Neumann neighborhood is much smaller than that with the Moore neighborhood; yet the latter reach equilibrium more easily (Fig. 2b). The spatial correlation with the Moore neighborhood of the metapopulation $I_{+}$limits to a value around 0.01 ; yet, the one with the von Neumann neighborhood limits to -0.01 (more regularly distributed). The spatial correlation of defectors is much more stable (less variance) than that of the cooperators (Fig. 2b). The spatial association also shows an interesting result (Fig. 2c). First, the association $A_{D / C}$ is below zero (negative association); while the association $A_{C / D}$ is larger than zero (positive association). Second, the dynamics of the association $A_{D / C}$ (standard deviation for $200<t<400$, std $=0.0244$ for $z=4$; std $=0.0075$ for $z=8$ ) is much more stable than those of $A_{C / D}(s t d=0.0474$ for $z=4$; std $=0.277$ for $z=8)$. Moreover, the association $A_{D / C}$ with the Moore neighborhood is significantly higher than that with the von Neumann neighborhood (Fig. $2 \mathrm{c}$; $t$-value $=54.87$, $d f=998, p<0.001)$.

Using pair approximation, we can study the spatial distribution and dynamics of metapopulations quantitatively. The dynamics and spatial patterns of cooperators and defectors in metapopulations can be profoundly influenced by the ratio of colonization to extinction coefficients $\alpha / \beta$, the temptation to defect $b$ and the neighboring structure $z$. When we overlap the global densities of cooperators and defectors on one graph, we can see the influence of the neighboring structures on coexistence in Fig. 3. First, the temptation-colonization plane can be divided into 

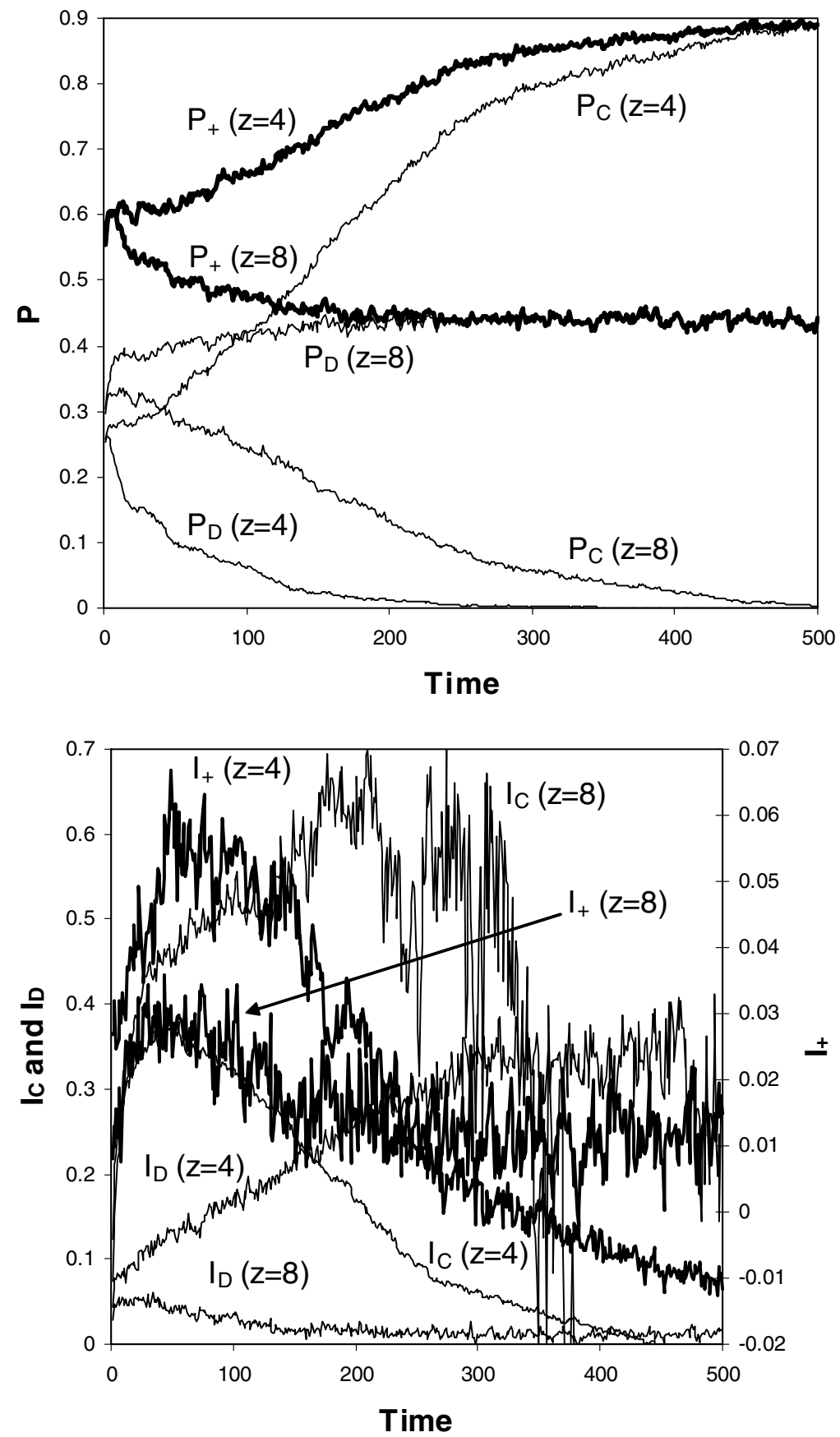

Fig. 2 Temporal dynamics of (a) occupancy (global density), (b) spatial correlation, and (c) spatial association in a spatial PDG $(80 \times 80$ patches $)$ with von Neumann and Moore neighborhood. Parameter values: $\alpha=0.7, \beta=0.2, b=1.6$. 


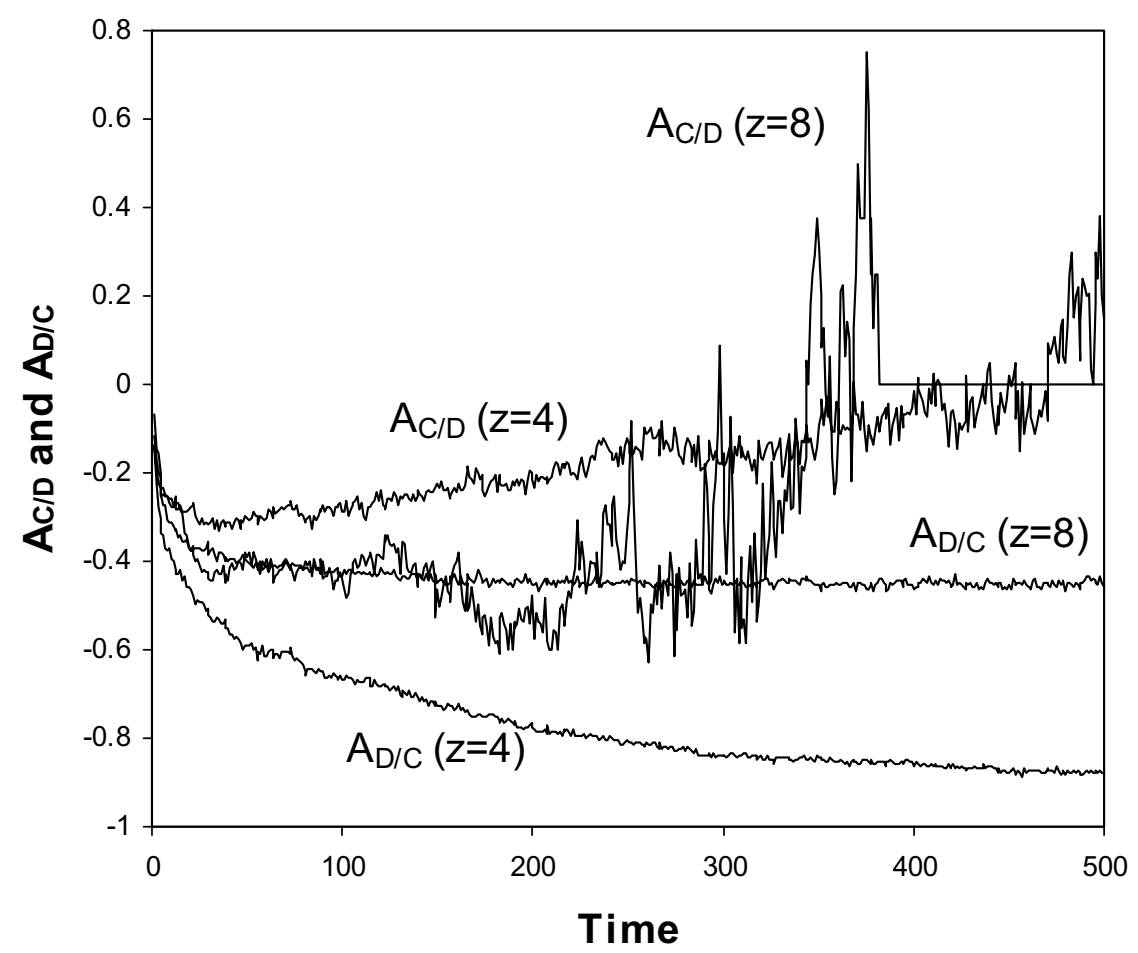

Fig. 2 Continued.

four parts: extinction (black region), pure cooperator (white region), coexistence of cooperator and defector (light gray) and pure defector (dark gray). With the increase of neighboring number (from low value of $z$ to the mean-field assumption, i.e. from a local-interacted population to a spatially well-mixed population), the region of pure cooperator will diminish and the region of pure defector will expand (Fig. 3). It implies that the mean-field approximation might underestimate the cooperator density and that the high connectivity of habitat patches will benefit defectors. Cooperators can even survive with the low colonization coefficient $\alpha<\beta$ in a highly local interaction ( $z=2$ in Fig. 3 ) because cooperators can compensate for the extinction debt and survive by the rewards of cooperation. This compensation breaks down with the connectivity of patches (such as $z=16$ in Fig. 3) because the defector has a high probability of meeting a cooperator during its lifetime and seizes the rewards. Therefore, the average payoff per local population in a metapopulation will decrease in highly connected habitat. It is a balance between the mean payoff of the local population, determined by PDG, and the viability of the metapopulation, determined by the colonization-extinction process. Patch connectivity has the opposite effect on the payoff of the local population and the metapopulation viability.

By pair approximation (Eq. 5), we can also calculate the global density $P_{\Omega}$ that indicates the fraction of $\Omega$-state patches (i.e. the occupancy) (Fig. 4 ) and the spatial 
(a)

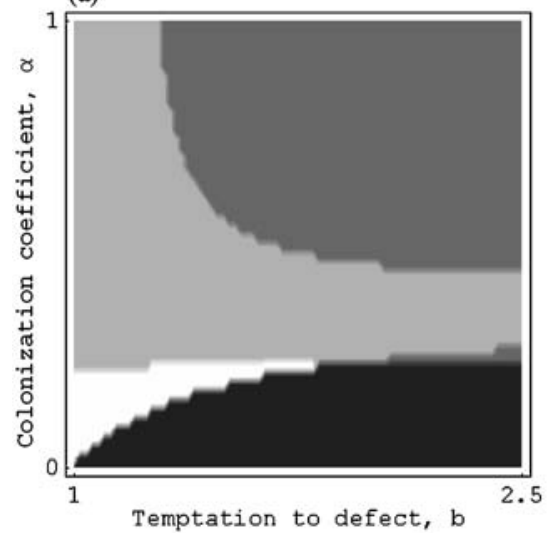

(c)

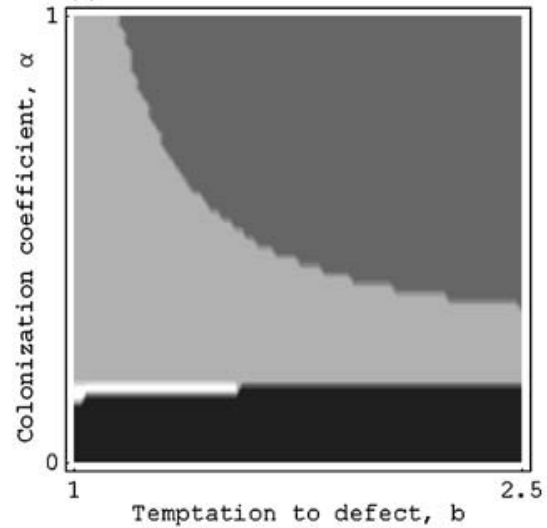

(b)

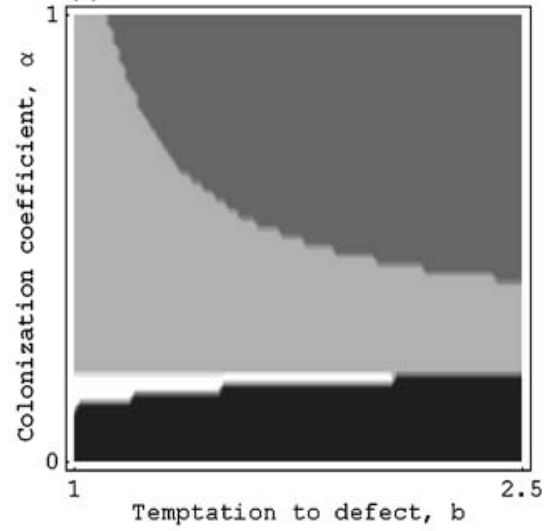

(d)

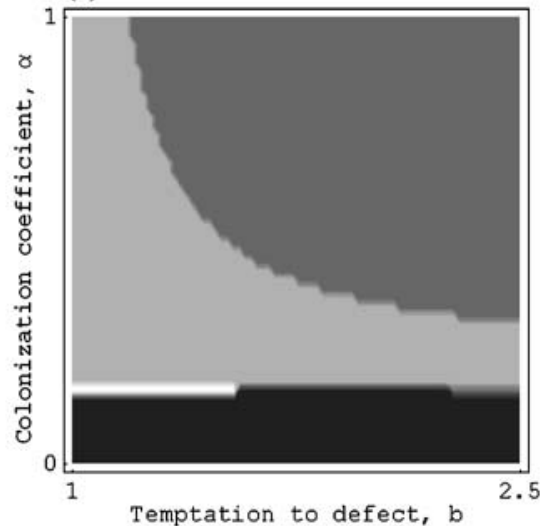

Fig. 3 The influence of the number of neighboring patches $(z)$ on spatial PDG. Regions of extinction (black), pure cooperator (white), coexistence of cooperator and defector (light gray) and pure defector (dark gray) in the temptation-colonization phase plane are plotted under different neighborhood $z \cdot z=2$ (a), 4(b), 8(c), and 16(d), respectively. Parameter values: $\beta=0.2$.

correlation $I_{\Omega}$ that indicates the contagiousness of the spatial distribution of $\Omega$ strategy local populations (Fig. 5), and the spatial association $\mathrm{A}_{\Omega / \Omega^{\prime}}$ that indicates the attraction of $\Omega^{\prime}$-strategy populations to $\Omega$-strategy ones (Fig. 6). Since the solutions to Eq. (5) are too complicated, we show these densities or probabilities under a fixed extinction coefficient $(\beta=0.2)$ in Figs. 4, 5, and 6. As for cooperators, the global density $P_{C}$ decreases with the number of neighboring patches $z$ (if $z \geq 4$; Fig. 4a) and with the temptation to defect $b$ (Fig. 4b). The global density of defectors $P_{D}$ increases with the colonization coefficient $\alpha$ (Fig. 4c, d) as well as with temptation to defect $b$ (Fig. 4d). However, the effect of patch neighborhood $z$ on $P_{D}$ is not significant (Fig. $4 \mathrm{c}$ ) ( $t$-test of $P_{D}$ for $z=2$ and $z=30 ; t$-value $=0.624$, $d f=98, p=0.534)$. As for the metapopulation size $P_{+}\left(P_{+}=P_{C}+P_{D}\right)$, the effect of $z$ is not significant for high colonization rate ( $t$-test of $P_{+}$for $0.5 \leq \alpha \leq 1$; $t$-value $=0.224, d f=48, p=0.823)$, but is significant when $z(2 \leq z \leq 4)$ and $\alpha$ 

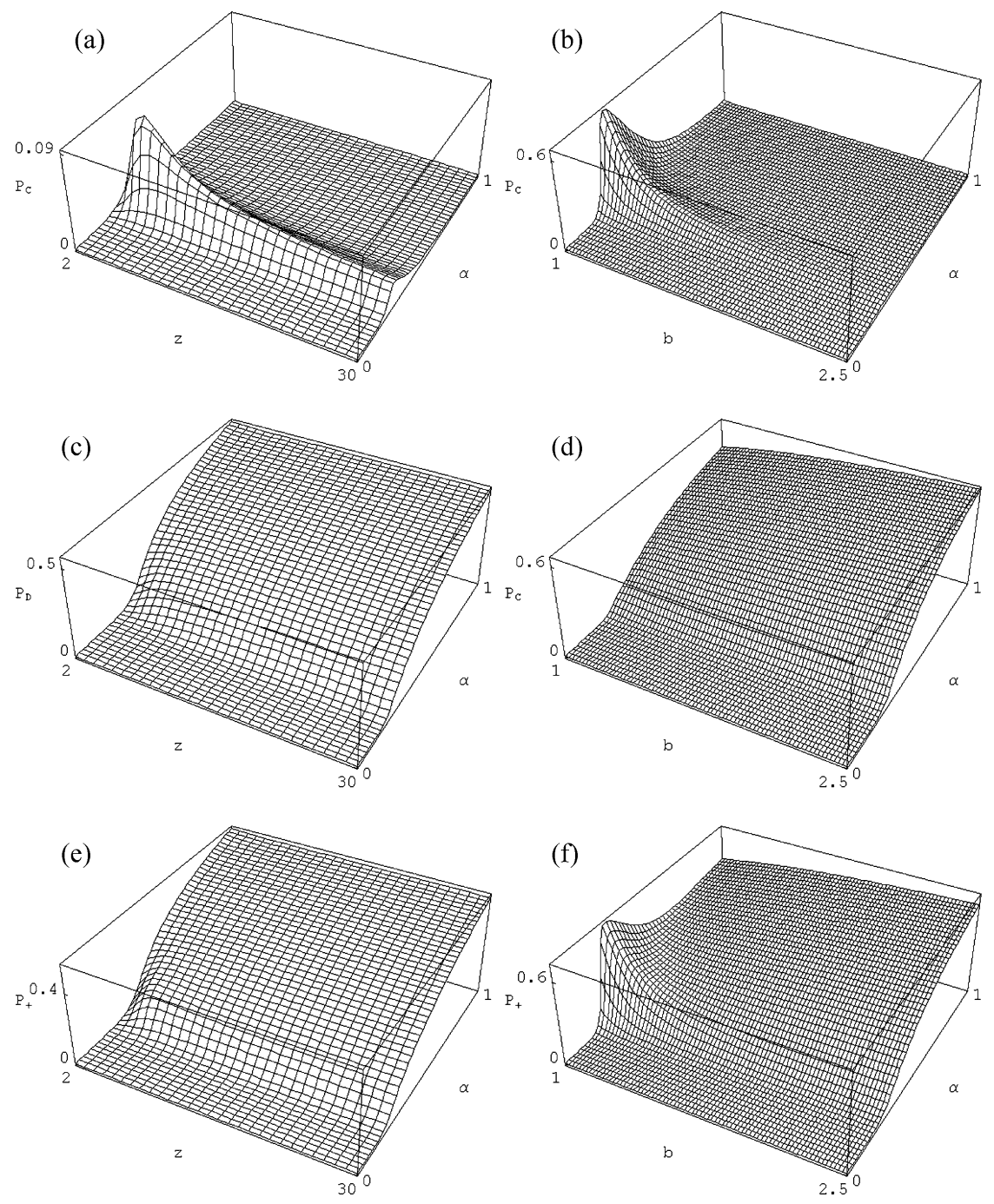

Fig. 4 Influence of the number of neighboring patches $(z)$, the temptation to defect $(b)$ and the colonization coefficient $(\alpha)$ on the occupancy of the cooperators $P_{C}(\mathrm{a}, \mathrm{b})$, the defectors $P_{D}(\mathrm{c}, \mathrm{d})$ and the metapopulations $P_{+}$(e, f). Parameter values: $\beta=0.2 ;(\mathrm{a}, \mathrm{c}, \mathrm{e}) b=1.4 ;(\mathrm{b}, \mathrm{d}, \mathrm{f}) z=4$.

(around $\beta$ ) are both small (Fig. 4e) ( $t$-test of $P_{+}$for $0.24 \leq \alpha \leq 0.4 ;$-value $=3.57$, $d f=14, p<0.01)$. When the temptation to defect is small $(b<1.5)$, an interesting phenomenon occurs (Fig. 4f): although the decrease of the colonization coefficient $\alpha$ is obviously harmful to the cooperators and defectors as well as the metapopulation (because their persistence relies on the colonization-extinction process), the metapopulation size $P_{+}$is amazingly increasing with the decrease of the colonization coefficient $\alpha$ (Fig. 4f). This counter-intuitive phenomenon only happens before the defectors are extinct. 

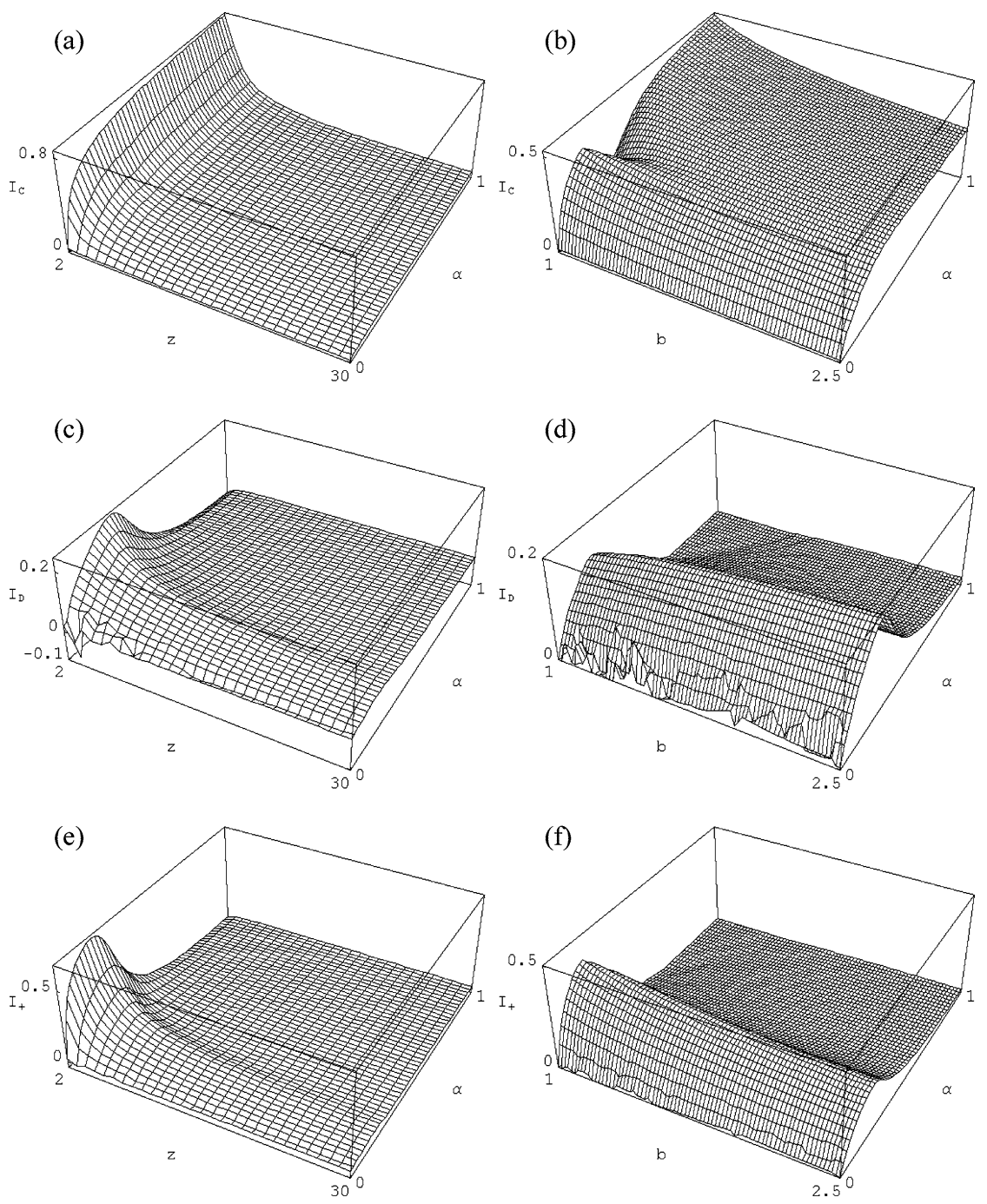

Fig. 5 Influence of the number of neighboring patches $(z)$, the temptation to defect $(b)$ and the colonization coefficient $(\alpha)$ on the spatial correlation of the cooperators $I_{C}(\mathrm{a}, \mathrm{b})$, the defectors $I_{D}$ (c, d) and the metapopulations $I_{+}(\mathrm{e}, \mathrm{f})$. Parameter values: $\beta=0.2 ;(\mathrm{a}, \mathrm{c}, \mathrm{e}) b=1.4 ;(\mathrm{b}, \mathrm{d}, \mathrm{f}) z=4$.

The spatial correlation of the cooperators $I_{C}$ declines rapidly with the increase of patch neighbors $z$ (Fig. 5a), but $I_{C}$ does not show a clear pattern with the increase of the colonization coefficient $\alpha$ or the temptation to defect $b$ (Fig. 5a, b). There is a threshold for the colonization coefficient $\alpha$ (equals 0.35 in Fig. 5). Above the threshold, the spatial correlation of the cooperators will increase at first and than decrease with the temptation to defect (Fig. 5b). If the colonization rate is large enough, spatial correlation $I_{C}$ will directly decrease with the temptation $b$ (Fig. 5b). The spatial correlation of the defectors $I_{D}$ declines with the number of 

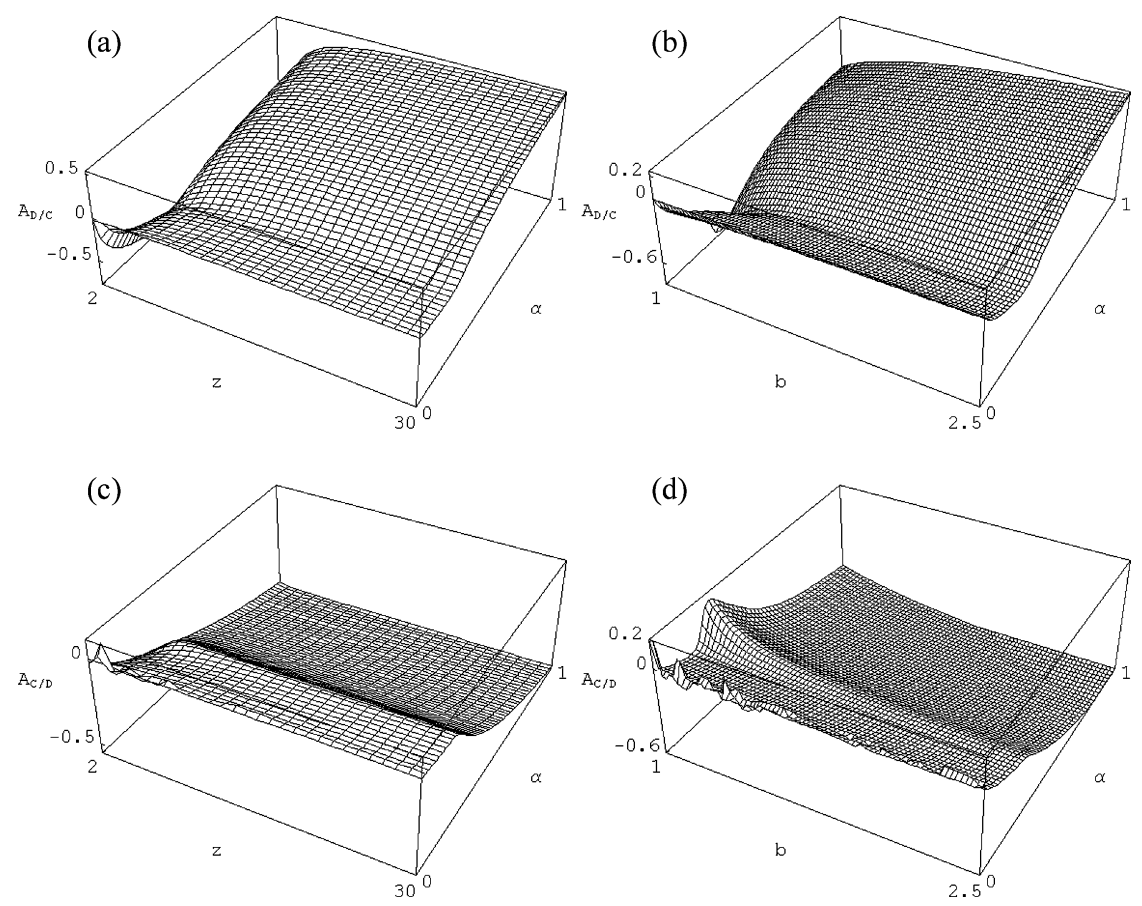

Fig. 6 Influence of the number of neighboring patches $(z)$, the temptation to defect $(b)$ and the colonization coefficient $(\alpha)$ on the spatial association between the cooperators and the defectors (a, b: $\left.A_{D / C} ; \mathrm{c}, \mathrm{d}: A_{C / D}\right)$. Parameter values: $\beta=0.2 ;(\mathrm{a}, \mathrm{c}) b=1.4 ;(\mathrm{b}, \mathrm{d}) z=4$.

patch neighbors $z$ (Fig. 5c), yet increases with the temptation to defect $b$ (Fig. 5d). Similarly, above the threshold $\alpha \approx 0.35$, with the increase of colonization coefficient $\alpha$, the spatial correlation of defectors $I_{D}$ will decrease. This is because the defectors try to avoid each other, leading to a more regular distribution in spatial habitat. As for the metapopulation, the spatial correlation $I_{+}=Q_{+/+}-P_{+}$declines with the increase of $z$ (Fig. 5e) and with the increase of $b$ (Fig. 5f). Above the threshold of $\alpha$, the spatial correlation of the metapopulation declines with $\alpha$ (but increases with $\alpha$ if $\alpha<0.35$ ) (Fig. 5e, f).

The spatial association $A_{\Omega / \Omega^{\prime}}\left(A_{\Omega / \Omega^{\prime}}=Q_{\Omega / \Omega^{\prime}}-Q_{\Omega^{\prime} / \Omega^{\prime}}\right)$ also shows a complicated pattern with the change of patch neighbors $z$, the colonization coefficient $\alpha$ and the temptation to defect $b$ (Fig. 6). With the increase of $z$ (Fig. 6a) and $b$ (Fig. 6b), the association $A_{D / C}$ increases from a negative value to a positive one, which indicates that more defectors will be found around the cooperators if we improve the connectivity of the patches or the temptation to defect. Above the threshold of $\alpha$, association $A_{D / C}$ will also increase with the colonization coefficient $\alpha$. The spatial association $A_{C / D}$ shows a general decline with the colonization coefficient $\alpha$ (Fig. 6c, d) and the temptation to defect $b$ (Fig. 6d). The effect of patch neighbors $z$ on the association $A_{C / D}$ is weak (Fig. 6c) ( $t$-test $A_{C / D}$ for $z=2$ and $z=30 ; t$-value $=1.29, d f=98, p=0.2$ ). It implies that fewer cooperators can 
be found if we raise the temptation to defect $b$ and the colonization coefficient $\alpha$ (Fig. 6c, d).

\section{Discussion}

We have built a spatial PDG in metapopulations. The colonization-extinction process was influenced by the payoffs in the spatial PDG. The influence of the colonization rate, the number of neighboring patches and the temptation to defect on the occupancy, spatial correlation and association were revealed. Cellular automaton shows a self-organized structure of the cooperators in the $2 \mathrm{D}$ spatial habitat (Fig. 1). The defectors cheat the cooperators only along the boundaries. However, the high payoffs of the cooperators guarantee the high success of colonization along the boundaries, which boycott the invasion of defectors. This boycott of cooperators to defectors in the spatial habitat might be an essential approach for survival.

The spatial version of PDG has two extensions. First, Huberman and Glance (1993) have introduced a continuous-time simulation to the model. They considered that the sites on the lattice are updated asynchronously, by randomly choosing a site and updating in each round. However, it is more plausible that the payoff of individuals only determines the probability that this individual will win in the next generation, instead of there being a deterministic updating rule (Nowak et al., 1994). The other extension is to incorporate spatial irregularities into the model. The individuals or sites are distributed randomly on a plane and only interact with neighbors who lie within some defined radius (Nowak et al., 1994). All of these extensions, either on time, the updating rule, or on space, do not change the basic result of coexistence of cooperators and defectors, although the details differs (Dieckmann et al., 2000). Our results also reinforce this finding. Contrasting the mean-field approximation Eq. (3) with the pair approximation Eq. (5), we can indicate that the necessary condition of the cooperation evolution is the local colonization of metapopulation.

The decline of the number of patch neighbors $z$ and the colonization coefficient $\alpha$ all contribute to the increase of the occupied patches by the cooperators, and therefore, favor the evolution of cooperation. Hui and Li (2003) divided habitat destruction into three types: habitat loss, patch isolation (that lead to dispersal cost and decrease in the colonization coefficient $\alpha$ ) and habitat decay (the decrease of the patch quality that increases the local extinction coefficient $\beta$ ). Zhang et al. (2005) have shown that patch isolation and habitat decay might help the cooperators finally win the spatial PDG. Because the decline in the number of patch neighbors $z$ and the colonization coefficient $\alpha$ indicates (1) the decline of patch connectivity and (2) the decline of dispersal ability, the results here also consistent with Zhang et al. (2005). Further studies show that other types of habitat destruction also improve the cooperative behavior in metapopulations (Zhang and Hui, in preparation). The environmental challenge to the metapopulation in fact gives a chance for the evolution of cooperation in the metapopulations. Altruistic behavior is in fact a strategic response of species to environmental stress. 
If there is only one behavior (either cooperators or defectors) in a metapopulation, the decline in the colonization rate is definitely harmful (i.e. the metapopulation size declines with habitat destruction; note the decline in colonization rate can be caused by habitat destruction, Hui and Li, 2003). However, the metapopulation with two behavior strategies can even increase its size with the declining colonization rate (Fig. 4f). This amazing result is, to some extent, analogous to Parrondo's paradox in game theory (Harmer and Abbott, 1999), which shows the possibility of winning by two losing gambling games. The reason here for the increase in metapopulation size is that the increase in the mean payoff of the cooperators $w_{C}$ overtakes the one of the defectors $w_{D}$, which in turn increases the colonization rate of the metapopulation, i.e. the compensation of cooperation rewards for extinction debt. It means that population size is not a consistent indicator of the environmental changes, especially for social species (McGeoch and Chown, 1998).

The temporary prosperity of the metapopulation in this work shows that it is not sufficient to monitor the environmental changes or habitat destruction by metapopulation size alone; the spatial structure is also needed. Wilson et al. (2004) indicated that declining species are expected to have sparse distribution because local extinctions cause retractions in range to optimal habitats (Brown et al., 1995; Johnson, 1998; Rodriguez and Delibes, 2002) or to locations that have been least affected by wide-acting extinction forces (Channell and Lomolino, 2000). Increasing species are expected to have more aggregated distributions, resulting from distance delimited colonization processes (Shigesada and Kawasaki, 1997). Results here show that those demonstrations were consistent with the spatial structure of defectors (Fig. 4c, d; Fig. 5c, d). However, the spatial structure of cooperators is not always consistent with those empirical conclusions (Fig. 4a, b; Fig. 5a, b). As a result, the spatial structure of metapopulations supports those conclusions in most ranges of the parameters, except in the region of temporary prosperity (Fig. 5f). The results here call for caution in predicting population trends by spatial structure, especially for species with multiple life history strategies (such as different morphologies or behaviors, etc.).

\section{Acknowledgements}

We are grateful to Z. Li, F. Zhang, X. Han, D.X. Yue, and two anonymous referees for their constructive comments and to B. Laniewski for her kind help with the English of this manuscript. This work was supported by the National Research Foundation of South Africa (GUN2053618) and the University of Stellenbosch.

\section{References}

Axelrod, R., Hamilton, W.D., 1981. The evolution of cooperation. Science 211, 1390-1396.

Brown, J.H., Mehlman, D.W., Stevens, G.C., 1995. Spatial variation in abundance. Ecology 76, 2028-2043.

Channell, R., Lomolino, M.V., 2000. Dynamic biogeography and conservation of endangered species. Nature 403, 84-86. 
Cole, L.C., 1957. The measurement of partial interspecific association. Ecology 38, 226-233.

Dieckmann, U., Law, R., Metz, J.A.J., 2000. The Geometry of Ecological Interactions: Simplifying Spatial Complexity. Cambridge University Press, New York.

Durrett, R., Levin, S., 1994. The importance of being discrete (and spatial). Theor. Popul. Biol. 46, 363-394.

Epstein, J.M., Axtell, R., 1996. Growing Artificial Societies. The Brookings Institution Press, Boston.

Fisher, R.A., 1930. The Genetical Theory of Natural Selection. Clarendon, Oxford.

Fortin, M.J., Dale, M.R.T., ver Hoef, J., 2002. Spatial analysis in ecology. In: El-Shaarawi, A.H., Piegorsch, W.W. (Eds.), Encyclopedia of Environmentrics. Wiley, New York, pp. 2051-2058.

Greig-Smith, P., 1964. Quantitative Plant Ecology, 2nd edn. Butterworths, London.

Hamilton, W.D., 1964. The genetical evolution of social behaviour. J. Theor. Biol. 7, 1-52.

Hanski, I., 1998. Metapopulation dynamics. Nature 396, 41-49.

Hanski, I., 1999. Metapopulation Ecology. Oxford University Press, Oxford.

Hanski, I., Gaggiotti, O.E., 2004. Ecology, Genetics, and Evolution of Metapopulations. Elsevier, Amsterdam.

Hanski, I., Zhang, D.Y., 1993. Migration, metapopulation dynamics and fugitive co-existence. J. Theor. Biol. 163, 491-504.

Harada, Y., Ezoe, H., Iwasa, Y., Matsuda, H., Sato, K., 1994. Population persistence and spatially limited social interaction. Theor. Popul. Biol. 48, 65-91.

Harada, Y., Iwasa, Y., 1994. Lattice population dynamics for plants with dispersing seeds and vegetative propagation. Res. Popul. Ecol. 36, 237-249.

Harmer, G.P., Abbott, D., 1999. Losing strategies can win by Parrondo's paradox. Nature 402, 864.

Harms, W., 1999. Biological altruism in hostile environments. Complexity 5(2), 23-28.

Hilborn, R.C., 2000. Chaos and Nonlinear Dynamics: An Introduction for Scientists and Engineers. Oxford University Press, New York.

Hoffmann, R., 2000. Twenty Years On: The Evolution of Cooperation Revisited. J. Artif. Soc. Soc. Simul. 3(2). <http://www.soc.surrey.ac.uk/JASSS/3/2/forum/1.html>

Huberman, B.A., Glance, N.S., 1993. Evolutionary games and computer simulations. Proc. Natl. Acad. Sci. USA 90, 7716-7718.

Hui, C., 2004. Spatial chaos of metapopulation incurred by Allee effect, overcrowding effect and predation effect. Acta Bot. Boreal.-Occident. Sin. 24, 370-383.

Hui, C., Li, Z., 2003. Dynamical complexity and metapopulation persistence. Ecol. Model. 164, 201-209.

Hui, C., Li, Z., 2004. Distribution patterns of metapopulation determined by Allee effects. Popul. Ecol. 46, 55-63.

Hui, C., Li, Z., Yue, D.X., 2004. Metapopulation dynamics and distribution, and environmental heterogeneity induced by niche construction. Ecol. Model. 177, 107-118.

Hui, C., McGeoch, M.A., Warren, M., 2006. A spatially explicit approach to estimating species occupancy and spatial correlation. J. Anim. Ecol. 75, 140-147.

Hui, C., Yue, D., 2005. Niche construction and polymorphism maintenance in metapopulations. Ecol. Res. 20, 115-119.

Hutchinson, G.E., 1961. The paradox of the plankton. Am. Nat. 95, 137-147.

Huxel, G.R., Hastings, A., 1999. Habitat loss, fragmentation, and restoration. Restor. Ecol. 7, 309-315.

Iwasa, Y., Sato, K, Nakashima, S. 1991. Dynamic modeling of wave regeneration (Shimagare) in subalpine Abies forests. J. Theor. Biol. 152, 143-158.

Johnson, C.N., 1998. Species extinction and the relationships between distribution and abundance. Nature 394, 272-274.

Katori, M., Konno, N., 1991. Upper bounds for survival probability of the contact process. J. Stat. Phys. 63, 115-130.

Kermer, J.E., Marquet, P.A., Johnson, A.R., 1998. Pattern formation in a patch occupancy metapopulation model: A cellular automata approach. J. Theor. Biol. 194, 79-90.

Levin, S.A., Grenfell, B., Hastings, A., Perelson, A.S., 1997. Mathematical and computational challenges in population biology and ecosystems sciences. Science 275, 334-343.

Levins, R., 1969. Some demographic and genetic consequences of environmental heterogeneity for biological control. Bull. Entom. Soc. Am. 15, 237-240. 
Lindgren, K., Nordahl, M.G., 1994. Cooperation and community structure in artificial ecosystems. Artif. Life 1, 15-37.

Matsuda, H., Ogita, A., Sasaki, A., Sato, K., 1992. Statistical mechanics of population: the lotkavolterra model. Prog. Theor. Phys. 88, 1035-1049.

McGeoch, M.A., Chown, S.L., 1998. Scaling up the value of bioindicators. Trends Ecol. Evol. 13, $46-47$.

McGlade, J.M., 1999. Advanced Ecological Theory: Principles and Applications. Blackwell, Oxford.

Nisbet, R.M., Gurney, W.S.C., 1982. Modelling Fluctuating Populations. Wiley, New York.

Nowak, M.A., Bonhoeffer, S., May, R.M., 1994. Spatial games and the maintenance of cooperation. Proc. Natl. Acad. Sci. USA 91, 4877-4881.

Nowak, M.A., May, R.M., 1992. Evolutionary games and spatial chaos. Nature 359, 826-829.

Nowak, M.A., Sigmund, K., 1993. A strategy of win-stay, lose-shift that outperforms tit-for-tat in the prisoner's dilemma game. Nature $364,56-58$.

Nowak, M.A., Sigmund, K., 2004. Evolutionary dynamics of biological games. Science 303, $793-$ 799.

Perry, J.N., Liebhold, A.M., Rosenberg, M.S., Dungan, J.L., Miriti, M., Jakomulska, A., CitronPousty, S., 2002. Illustrations and guidelines for selecting statistical methods for quantifying spatial pattern in ecological data. Ecography 25, 578-600.

Pielou, E.C., 1972. Measurement of structure in animal communities. In: Wiens, J.A. (Ed.), Ecosystem Structure and Function. Oregon State University Press, Corvallis, pp. 113-136.

Rodriguez, A., Delibes, M., 2002. Internal structure and patterns of contration I the geographic range of the Iberian Lynx. Ecography 25, 314-328.

Sato, K., Iwasa, Y., 2000. Pair approximation for lattice-based ecological models. In: Dieckmann, U., Law, R., Metz, J.A.J. (Eds.), The Geometry of Ecological Interactions: Simplifying Spatial Complexity. Cambridge University Press, Cambridge, pp. 341-358.

Sayama, H., 2004. Self-protection and diversity in self-replicating cellular automata. Artif. Life 10, $83-98$.

Schonfisch, B., 1997. Anisotropy in cellular automata. Biosystems 41, 29-41.

Shigesada, N., Kawasaki, K., 1997. Biological Invasions: Theory and Practice. Oxford University Press, Oxford.

Tainaka, K., 1993. Paradoxical effect in a three-candidate voter model. Phys. Lett. A 176, 303-306.

Tilman, D., 1994. Competition and biodiversity in spatially structured habitats. Ecology 75, 2-16.

Tilman, D., Kareiva, P., 1997. Spatial Ecology: The Role of Space in Population Dynamics and Interspecific Interactions. Princeton University Press, Princeton.

Trivers, R., 1971. The evolution of reciprocal altruism. Q. Rev. Biol. 46, 35-57.

Upton, G.J.G., Fingleton, B., 1985. Spatial Data Analysis by Example, vol. 1: Point Pattern and Quantitative Data. Wiley, New York.

Wilkinson, G.S., 1984. Reciprocal food sharing in the vampire bat. Nature 308, 181-184.

Williams, G.C., 1966. Adaptation and Natural Selection. Princeton University Press, Princeton.

Wilson, D.S., Sober, E., 1994. Re-introducing group selection to human behavioral sciences. Behav. Brain Sci. 17, 585-654.

Wilson, R.J., Thomas, C.D., Fox, R., Roy, D.B., Kunin, W.E., 2004. Spatial patterns in species distributions reveal biodiversity change. Nature 432, 393-396.

Zhang, F., Hui, C., Han, X., Li, Z., 2005. Evolution of cooperation in patchy habitat under patch decay and isolation. Ecol. Res. 20, 461-469. 